\title{
El apoyo psicosocial no prolonga la sobrevida pero mejora su calidad en mujeres con cáncer metastático de mama
}

The effect of group psychosocial support on survival in metastatic breast cancer. Goodwin PJ, Leszcz M, Ennis M y col. N Engl J Med. 2001, December 13,345,1719 1726.

\section{Objetivo}

Evaluar si la terapia de apoyo psicosocial prolonga la sobrevida en mujeres con cáncer metastático de mama.

En segundo lugar evaluar la adaptación psicológica de las pacientes, su experiencia frente al dolor y su calidad de vida.

\section{Diseño}

Ensayo clínico, aleatorizado con un seguimiento promedio de 15 meses.

\section{Lugar}

Estudio m ulticéntrico realizado en siete centros de Canadá.

\section{Pacientes}

235 mujeres con cáncer matastásico de mama

\section{Intervención}

Se aleatorizó a las pacientes en dos grupos, uno de ellos recibió intervención del grupo psicosocial $(n=158)$ y el otro no, grupo control $(n=77)$.Se estratificó de acuerdo al centro y a la presencia o ausencia de metástasis viscerales.

\section{Medición de Resultados Principales}

El resultado principal fue la medición de la sobrevida total por el método de análisis de sobrevida de Kaplan Meier.*

\section{Resultados Principales}

La sobrevida media fue de 17.9 meses en el grupo que recibió apoyo psicosocial y de 17.6 meses en el grupo control ( $p: 0.72$ ), no existiendo diferencias significativas en la sobrevida total.

En el modelo multivariable se analizó la presencia o ausencia de receptores de progesterona $(p=0.01)$, la presencia 0 ausencia de receptores de estrógeno ( $p: 0.71$ ), el tiempo transcurrido desde la primera metástasis a la randomización ( $\mathrm{p}: 0.50$ ), edad al momento del diagnóstico ( $p: 0.85)$, estadío ganglionar al diagnóstico ( $p: 0.40)$ y si recibió o no-quimioterapia adyuvante ( $p: 0.11)$, ninguna de ellas fue significativa al analizar los resultados finales.

Los scores obtenidos para trastornos totales del humor al año después de la randomización fueron significativamente más bajo en el grupo que recibió la intervención psicosocial comparado con el grupo control ( $p: 0.02)$.

Al evaluar el dolor y el sufrimiento por medio de una escala visual análogica., las mujeres que tuvieron apoyo psicosocial presentaron menos empeoramiento del dolor en el curso de un año que las del grupo control ( $p: 0.04)$.No hubo diferencias significativas con respecto a la evaluación del sufrimiento.

\section{Conclusiones}

El aporte de un grupo de apoyo psicosocial al tratamiento standard de las pacientes con cáncer metastático de mama no tiene influencia en la sobrevida.

\section{Comentario}

Las situaciones de stress que se presentan en los pacientes a los que se les diagnostica un cáncer son de gran envergadura, ya que muchas veces el diagnóstico amenaza la continuidad de la vida ${ }^{1}$. Las intervenciones psicosociales se basan en varios puntos:una intervención educativa y reestructurativa (conocimiento sobre los tratamientos y manejo de la ansiedad, por ejemplo), soporte social, permitirse la exteriorización de emociones, discutir o confrontar la ansiedad sobre la muerte, reordenar prioridades de vida, estimular el soporte familiar, mejorar la comunicación con sus médicos y entrenamiento en técnicas de relajación muscular progresiva, meditación, etc. 2, 3 .

En este estudio se encontró mejoría de síntomas como el dolor en las pacientes que fueron tratadas por el grupo de soporte psicosocial y esto mismo fue comprobado por otros grupos de trabajo y en otros síntomas como nauseas y vómitos 2.También se observó la disminución de trastornos del humor como ansiedad y depresión al año de la intervención.

Las intervenciones psicosociales en pacientes enfermos incluyen también una rápida recuperación después de una cirugía, una disminución del tiempo de estadía en el hospital y disminución del stress en las visitas de los pacientes a sus médicos de cabecera 2 . Spiegel y col. 2 realizaron un estudio con mujeres con cáncer de mama metastático y obsenaron que 50 mujeres con este diagnóstico y tratadas por grupos de soporte psicosocial presentaban una sobrevida mayor, (promedio de 18 meses más), con respecto a un grupo control de 36 pacientes. El presente estudio de Goodwin y col evaluó las mismas intervenciones psicosociales (el personal dedicado a ellas fue asesorado por Spiegel y col), pero con una población mayor (235 pacientes) y los resultados no fueron los mismos. No parece estar muy claro si la intervención psicosocial aumenta o no la sobrevida 5 . Hay pocos trabajos publicados al respecto y las opiniones están divididas 2,4 . Es importante destacar una desventaja del estudio de Spiegel que es la escasa cantidad de pacientes.

Conclusión: Necesitamos mejor evidencia para determinar si estas intervenciones benefician o no la sobrevida total de pacientes con cáncer y poder identificar a la población que más las necesitan. De todas maneras es importante destacar el efecto que este tipo de terapia parece lograr en la calidad de esa sobrevida.

\section{Dra. Adriana Velasco Leiva [ Cuidados Paliativos.Hospital Italiano de Buenos Aires ]}

\author{
Referencias \\ 1. Holland J, Rowland J Handbook of Psychooncology, Psychologic Care of the Patient with CanceNew York, Oxford University, 1990. \\ 2. Spiegel D, Diamon S Psychosocial Intenentions. Thirty Fourth Annual Meeting of A.SC.O., 1998.
3. Fawzy I, Fawzy N, Canada A Psychoeducational Intervention Programs for Patients with Cancerhirty Fourth Annual Meeting of A.SC.O., 1998. \\ 3. Fawzy I, Fawzy N, Canada A Psychoeducational Intervention Programs for Patients with Cancerthirty Fourth Annual Meeting of A.SC.O., 1998. \\ 5. De Vita V, Hellman S, Rosenberg S CancerPrinciples and Practice of Oncology 6th edition, 2001.
}

